\title{
Preparation of 2-Arylquinolines from 2-Arylethyl Bromides and Aromatic Nitriles with Magnesium and $\mathrm{N}$-Iodosuccinimide
}

\author{
Hiroki Naruto \\ Hideo Togo* (D) \\ Graduate School of Science, Chiba University, Yayoi-cho 1-33, \\ Inage-ku, Chiba 263-8522, Japan \\ togo@faculty.chiba-u.jp
}

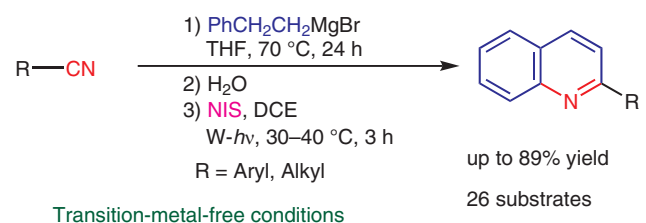

with easily available reagents
Received: 18.11.2019

Accepted after revision: 19.12.2019

Published online: 23.01 .2020

DOI: 10.1055/s-0039-1691642; Art ID: ss-2019-f0638-op

Abstract Treatment of 2-arylethylmagnesium bromides, prepared from 2-arylethyl bromides and magnesium, with aromatic nitriles, followed by reaction with water and then with $N$-iodosuccinimide under irradiation with a tungsten lamp, gave the corresponding 2-arylquinolines in good to moderate yields under transition-metal-free conditions. 2-Alkylquinolines could be also obtained in moderate yields by the same procedure with 2-arylethyl bromides, magnesium, aliphatic nitriles bearing a secondary alkyl group, and $\mathrm{N}$-iodosuccinimide.

Key words quinolines, 2-arylethyl bromides, aromatic nitriles, Grignard reaction, $\mathrm{N}$-iodosuccinimide

Quinolines are one of the most important nitrogencontaining heteroaromatics, because of their potent biological activities, such as antimalarial, antibacterial, anti-inflammatory, and anticancer activities. ${ }^{1}$ As typical examples (Figure 1), quinine (natural product) and chloroquine (synthesized product) are antimalarials. ${ }^{2}$

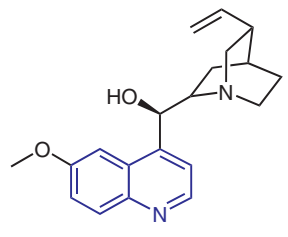

Quinine

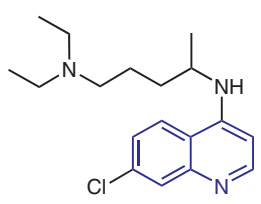

Chloroquine
Figure 1 Typical quinolines possessing antimalarial activity

Synthetic studies of the quinoline core have been actively carried out. ${ }^{3}$ Conventionally, the quinoline core has been prepared by named reactions, such as the Skraup synthesis, the Friedländer synthesis, the Combes synthesis, and the Conrad-Limpach synthesis, among others. ${ }^{4}$ Recent reports of the preparation of the quinoline core are as follows: ${ }^{5}$ the preparation of 2-aryl-4-methylquinolines with hydrazones of $o$-aminoacetophenone and ethynylarenes in the presence of $\left[\mathrm{RhCp}^{*} \mathrm{Cl}_{2}\right]_{2},{ }^{5 a}$ the preparation of 2-arylquinolines with anilines and aromatic aldehydes in the presence of $\mathrm{FeCl}_{3}$ in nitroethane; ${ }^{5 b}$ the preparation of 2-aryl-3-[(trifluoromethyl)sulfanyl]quinolines with 0 -alkynylbenzyl azides, $\mathrm{Na}_{2} \mathrm{~S}_{2} \mathrm{O}_{8}$, and $\mathrm{AgSCF}_{3},{ }^{5 \mathrm{c}}$ the preparation of 2-amino-3arylquinolines with $o$-aminobenzyl alcohols and $\alpha$-arylacetonitriles in the presence of $\mathrm{Mn}(\mathrm{I})-\mathrm{NNS}$ complex; ${ }^{5 \mathrm{~d}}$ the preparation of 4-amino-2-(difluoromethyl)-3-(methoxycarbonyl)quinolines with 0 -aminobenzonitriles and methyl 4,4-difluorobut-2-ynoate; ${ }^{5 e}$ the preparation of 4phenylquinolines with anilines, $\alpha$-alkynyl esters, and phenylacetylene in the presence of AgOTf; 5 the preparation of 2 -aroyl-3,4-diarylquinolines with $\alpha$-( $N$-arylamino)acetophenones, 1,1-diarylethenes, and di-tert-butyl peroxide in the presence of $\mathrm{Cu}(\mathrm{OTf})_{2}, 5 \mathrm{~g}$ the preparation of 4 -(acylmethyl)-2-aminoquinolines with $\beta$-(o-aminophenyl)- $\alpha, \beta$-ynones and ynamides in the presence of $\mathrm{Au}(\mathrm{I}){ }^{5 \mathrm{~h}}$ the preparation of 2-aminoquinolines with $\mathrm{N}$-acyl-o-alkynylanilines and isocyanides in the presence of $\mathrm{Pd}(\mathrm{OAc})_{2} ;{ }^{5 i}$ the preparation of 2aryl-3-tosylquinolines with anthranils and $\mathrm{N}$-tosylhydrazones in the presence of $\mathrm{Cu}(\mathrm{OAc})_{2}$ and AgOTf; 5 je the preparation of 6-(aryldiazenyl)-3-iodoquinolines with (o-aminoaryl)propargyl alcohols, aryldiazonium salts, and $\mathrm{I}_{2} ;{ }^{5 \mathrm{k}}$ the preparation of 3-aryl-2-(arylsulfonyl)quinolines with 0 alkynylisocyanobenzenes and $p-\mathrm{TsNa}^{5}$; and the preparation of 2,4-diarylquinolines with 0 -aminobenzyl alcohols and alcohols in the presence of $\mathrm{Mn}(\mathrm{I})-\mathrm{PNP}$ complex. ${ }^{5 \mathrm{~m}}$

On the other hand, recently, synthetic uses of the iminyl radical (nitrogen-centered radicals) have become popular, particularly in the preparation of nitrogen-containing heterocyclic compounds such as dihydropyrroles and phenanthridines. ${ }^{6}$ As synthetic uses of iminyl radicals formed from ketimines and molecular iodine, we reported a one-pot 
preparation of 6-arylphenanthridines by the treatment of $o$-cyanobiaryls with aryllithiums, followed by reaction with water and then with molecular iodine at $60{ }^{\circ} \mathrm{C},{ }^{7}$ and of 2arylquinolines by the treatment of 3-arylpropionitriles with aryllithiums, followed by reaction with water and then with $N$-iodosuccinimide (NIS) under irradiation with a tungsten lamp (Scheme 1, eq 1). ${ }^{8}$ The latter method is suitable for the preparation of 2-arylquinolines bearing an alkyl group, such as methyl, ethyl, or isopropyl, at the 3-position. However, it cannot be practically used for the preparation of 2-arylquinolines due to the occurrence of $\alpha$-proton abstraction of 3-arylpropionitriles by aryllithiums in the $1^{\text {st }}$ reaction step. Herein, we report the transformation of 2arylethyl bromides into 2-arylquinolines by the treatment with magnesium and then with aromatic nitriles, followed by reaction with water and then with NIS under irradiation with a tungsten lamp (Scheme 1, eq 2).
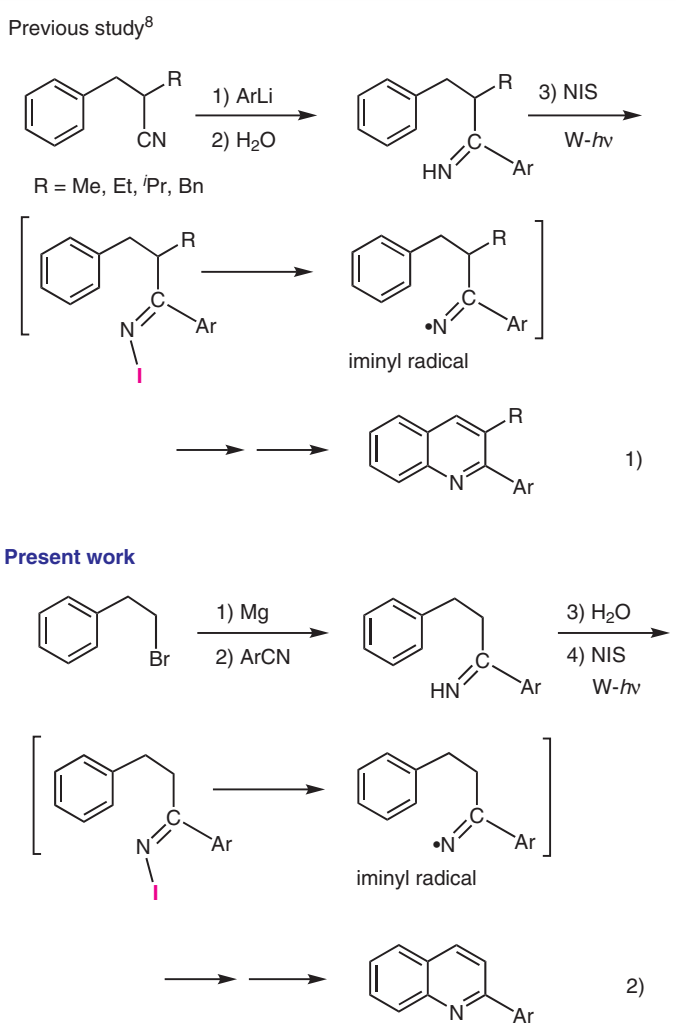

Scheme 1 Preparation of 2-arylquinolines via $\mathrm{N}$-iodoimines and iminyl radicals

First, to understand the reactivity of 2-arylethylmagnesium bromides toward aromatic nitriles and secondary aliphatic nitriles, the reactions of 2-phenylethylmagnesium bromide with $p$-tolunitrile (1A) and with isobutyronitrile (1X) at $70{ }^{\circ} \mathrm{C}$ for 6 and 24 hours, followed by aqueous $\mathrm{HCl}$ hydrolysis to form the corresponding ketones $\mathbf{2} \mathbf{A}^{\prime}$ and $\mathbf{2 X}$ ', respectively, were carried out (Scheme 2 , eq 1 and 2 ). The results suggest that warming treatment at $70{ }^{\circ} \mathrm{C}$ for 24 hours is better, and the maximum yields of ketones $\mathbf{2} \mathbf{A}^{\prime}$ and $\mathbf{2 X}^{\prime}$ with $p$-tolunitrile (1A) and isobutyronitrile (1X) were $94 \%$ and $57 \%$, respectively, due to the partial occurrence of $\alpha$-proton abstraction from isobutyronitrile by 2-phenylethylmagnesium bromide. An excess amount of 2-phenylethylmagnesium bromide was used as the reactivity of 2-phenylethylmagnesium bromide toward $p$-tolunitrile and toward isobutyronitrile was not sufficiently high and warming conditions at $70{ }^{\circ} \mathrm{C}$ for 24 hours were required.
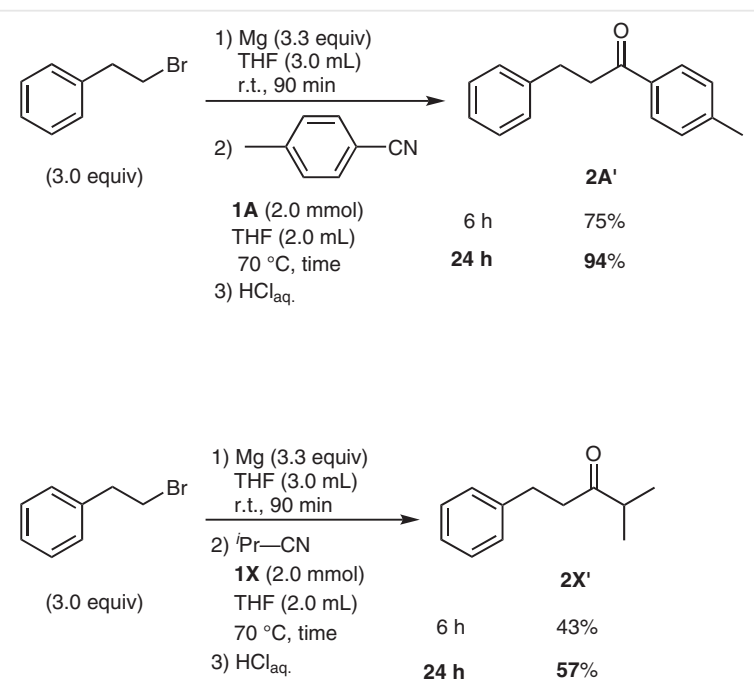

Scheme 2 Formation of ketones $\mathbf{2} \mathbf{A}^{\prime}$ and $\mathbf{2} \mathbf{X}^{\prime}$ with 2-phenylethylmagnesium bromide and nitriles 1

Then, treatment of 2-phenylethylmagnesium bromide, prepared from 2-phenylethyl bromide (3.0 equiv) and magnesium (3.3 equiv) in THF $(5.0 \mathrm{~mL})$, and $p$-tolunitrile $(\mathbf{1 A}$, $2.0 \mathrm{mmol}$ ) at $70{ }^{\circ} \mathrm{C}$ for 24 hours ( $\left.1^{\text {st }} \mathrm{step}\right)$, followed by the addition of water ( $5.0 \mathrm{~mL}, 2^{\text {nd }}$ step), gave $p$-methylphenyl 2phenylethyl ketimine (2A). After rapid extraction of ketimine 2A with chloroform and removal of the solvent, ketimine 2A was treated with NIS (3.0 equiv) in 1,2-dichloroethane (DCE, $6.0 \mathrm{~mL}$ ) under irradiation with a tungsten lamp (300 W) for 3 hours in the temperature range of 30$40{ }^{\circ} \mathrm{C}$ ( $3^{\text {rd }}$ step) to give 2-(4-methylphenyl)quinoline (3A) in $73 \%$ yield, as shown in Table 1, entry 1 . After the same reactions in the $1^{\text {st }}$ reaction step, treatment of the reaction mixture with methanol $(3.0 \mathrm{~mL})$ and then DCE $(6.0 \mathrm{~mL})$, followed by reaction with NIS (3.0 equiv) under irradiation with a tungsten lamp, gave $\mathbf{3 A}$ in only $21 \%$ yield (entry 2 ). Thus, the one-pot preparation of 2-(4-methylphenyl)quinoline (3A) from 2-phenylethylmagnesium bromide and $p$ tolunitrile (1A) was not effective because NIS was consumed by remaining magnesium and methanol. Under the same procedure and conditions as those of entry 1 ( $1^{\text {st }}$ and $2^{\text {nd }}$ steps), treatment of ketimine 2A with NIS (3.5 equiv) in DCE under irradiation with a tungsten lamp for 3 and 6 hours generated quinoline $\mathbf{3 A}$ in $82 \%$ and $81 \%$ yield, respectively (entries 3 and 4), although the same treatment with 
NIS (4.0 equiv) slightly reduced the yield of $\mathbf{3 A}$ (entry 5 ). Under the same procedure and conditions as those of entry 3 , a solution of ketimine $\mathbf{2 A}$ and NIS was irradiated with an LED lamp (13.6 W) and a $40 \mathrm{~W}$ tungsten lamp, instead of a $300 \mathrm{~W}$ tungsten lamp, in the $3^{\text {rd }}$ reaction step to give $\mathbf{3 A}$ in $71 \%$ and $67 \%$ yield, respectively (entries 6 and 7). Moreover, room light instead of irradiation with a $300 \mathrm{~W}$ tungsten lamp was not effective at all (entry 8). On the other hand, warming treatment of the mixture under dark conditions in the $3^{\text {rd }}$ reaction step gave 2-(4-methylphenyl)quinoline (3A) in 66\% yield (entry 9). Thus, entry 3 showed the best result. When 1,3-diiodo-5,5-dimethylhydantoin (DIH, 1.75 equiv) was used instead of NIS under the same procedure and conditions as those of entry 3, 3A was obtained in $82 \%$ yield again (entry 10 ). On the other hand, warming treatment of a solution of ketimine $\mathbf{2 A}$ with $\mathrm{I}_{2}$ in the presence of $\mathrm{K}_{2} \mathrm{CO}_{3}$ at $70^{\circ} \mathrm{C}$ for 3 hours, and also irradiation treatment of ketimine $\mathbf{2 A}$ with $\mathrm{N}$-bromosuccinimide (NBS) or $\mathrm{N}$-chlorosuccinimide (NCS) with a tungsten lamp for 3 hours at 30$40{ }^{\circ} \mathrm{C}$, did not generate $\mathbf{3 A}$ at all (entries $11-13$ ), and $p$ methylphenyl 2-phenylethyl ketone $\left(\mathbf{2 A}^{\prime}\right)$, a hydrolyzed compound of ketimine $\mathbf{2 A}$ by quenching the reaction mixture with aqueous $\mathrm{Na}_{2} \mathrm{SO}_{3}$, was obtained in more than $80 \%$ yield. Thus, NIS and DIH were effective for the present $3^{\text {rd }}$ reaction step. When the $3^{\text {rd }}$ reaction step was carried out in the presence of 2,2,6,6-tetramethylpiperidine 1-oxyl (TEMPO, 1.5 equiv) or 2,6-di-tert-butyl-p-cresol (BHT, 1.5 equiv), $3 \mathrm{~A}$ was not obtained at all (entries 14 and 15).

Based on those results, 2-phenylethylmagnesium bromide prepared from 2-phenylethyl bromide (3.0 equiv) and magnesium (3.3 equiv) in THF $(5.0 \mathrm{~mL})$ was treated with aromatic nitriles 1B-10 $(2.0 \mathrm{mmol})$, bearing a o-methylphenyl (B), $m$-methylphenyl (C), phenyl (D), p-tert-butylphenyl (E), 3,5-dimethylphenyl (F), p-methoxyphenyl (G), $p$-fluorophenyl $(\mathbf{H}), p$-chlorophenyl (1), $m$-bromophenyl $(\mathbf{J}), p$ bromophenyl (K), $p$-(trifluoromethyl)phenyl (L), $\beta$-naphthyl $(\mathbf{M}), p$-biphenyl $(\mathbf{N})$, or $p$-phenoxyphenyl (0) group, at $70{ }^{\circ} \mathrm{C}$ for 24 hours ( $1{ }^{\text {st }}$ step), and then with water $(5.0 \mathrm{~mL}$, $2^{\text {nd }}$ step) to give aryl 2-phenylethyl ketimines 2 . After extraction of ketimines $\mathbf{2}$ with chloroform and removal of the solvent, ketimines 2 were treated with NIS (3.5 equiv) in DCE $(6.0 \mathrm{~mL})$ under irradiation with a tungsten lamp (300 $\mathrm{W})$ for 3 hours in the temperature range of $30-40{ }^{\circ} \mathrm{C}\left(3^{\text {rd }}\right.$ step) to form 2-arylquinolines $\mathbf{3 B}-\mathbf{3 0}$ in good to moderate yields, except 3B in 35\% yield (Scheme 3). As a gram-scale experiment, when 2-phenylethylmagnesium bromide was treated with benzonitrile (1D, $10 \mathrm{mmol})$ under the same procedure and conditions, 2-phenylquinoline (3D) was obtained in $71 \%$ yield (Scheme 3). Treatment of 2-phenylethylmagnesium bromide with aromatic nitriles 1P and $1 \mathbf{Q}$ bearing an acetal and MOM group under the same procedure and conditions generated the corresponding 2arylquinolines $\mathbf{3 P}$ and $\mathbf{3 Q}$ in a moderate and good yield, respectively.
Table 1 Optimization of the Reaction Conditions for Compound 3A

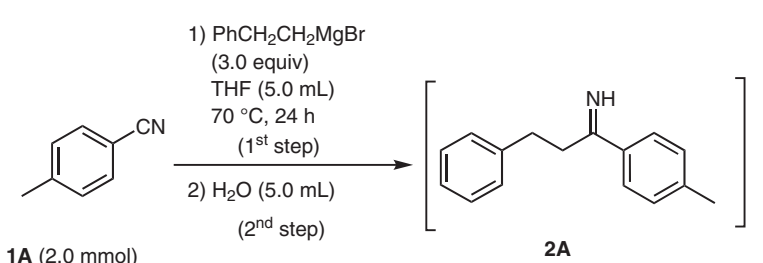

1 A $(2.0 \mathrm{mmol})$

$2 A$

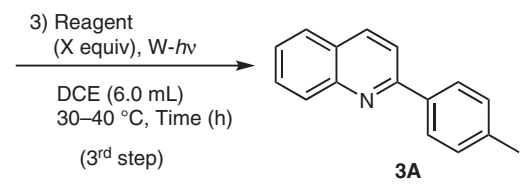

\begin{tabular}{|c|c|c|c|c|}
\hline Entry & Reagent & X (equiv) & Time (h) & Yield (\%) \\
\hline 1 & NIS & 3.0 & 3 & 73 \\
\hline $2^{\mathrm{a}}$ & NIS & 3.0 & 3 & 21 \\
\hline 3 & NIS & 3.5 & 3 & 82 \\
\hline 4 & NIS & 3.5 & 6 & 81 \\
\hline 5 & NIS & 4.0 & 3 & 78 \\
\hline $6^{\mathrm{b}}$ & NIS & 3.5 & 3 & 71 \\
\hline $7^{c}$ & NIS & 3.5 & 3 & 67 \\
\hline $8^{d}$ & NIS & 3.5 & 3 & 5 \\
\hline $9^{e}$ & NIS & 3.5 & 3 & 66 \\
\hline 10 & $\mathrm{DIH}$ & 1.75 & 3 & 82 \\
\hline $11^{f}$ & $\mathrm{I}_{2}$ & 3.5 & 3 & 0 \\
\hline 12 & NBS & 3.5 & 3 & 0 \\
\hline 13 & NCS & 3.5 & 3 & 0 \\
\hline $14^{9}$ & NIS & 3.5 & 3 & 0 \\
\hline $15^{\mathrm{h}}$ & NIS & 3.5 & 3 & 0 \\
\hline
\end{tabular}

${ }^{\mathrm{a}} \mathrm{MeOH}(3.0 \mathrm{~mL})$ was used instead of $\mathrm{H}_{2} \mathrm{O}$, and the obtained mixture was directly treated with NIS under irradiation with a tungsten lamp.

${ }^{b}$ A white LED lamp (13.6 W) was used, instead of a $300 \mathrm{~W}$ tungsten lamp.

c A $40 \mathrm{~W}$ tungsten lamp was used, instead of a $300 \mathrm{~W}$ tungsten lamp.

d Room light (fluorescent lighting, $32 \mathrm{~W}$ ) was used, instead of a $300 \mathrm{~W}$ tungsten lamp.

e The $3^{\text {rd }}$ step reaction was carried out at $70{ }^{\circ} \mathrm{C}$ under dark conditions.

${ }^{f}$ In the $3^{\text {rd }}$ step reaction, $\mathrm{K}_{2} \mathrm{CO}_{3}(1.5$ equiv) was added.

9 In the $3^{\text {rd }}$ step reaction, TEMPO (1.5 equiv) was added.

${ }^{\mathrm{h}}$ In the $3^{\text {rd }}$ step reaction, BHT (1.5 equiv) was added.

When 2-( $p$-methylphenyl)ethylmagnesium bromide, 2( $p$-chlorophenyl)ethylmagnesium bromide, and 2-( $p$-methoxyphenyl)ethylmagnesium bromide were used instead of 2-phenylethylmagnesium bromide in the reaction with benzonitrile (1D) under the same procedure and conditions, 7-methyl-2-phenylquinoline (3R), 7-chloro-2phenylquinoline (3S), and 7-methoxy-2-phenylquinoline (3T) were obtained in good to moderate yields (Scheme 3). Treatment of 2-phenyl-1-propylmagnesium bromide with benzonitrile and $p$-tolunitrile under the same procedure and conditions generated 4-methyl-2-phenylquinoline (3U) and 4-methyl-2-(4-methylphenyl)quinoline (3V), respectively, in moderate yields. On the other hand, treatment of 


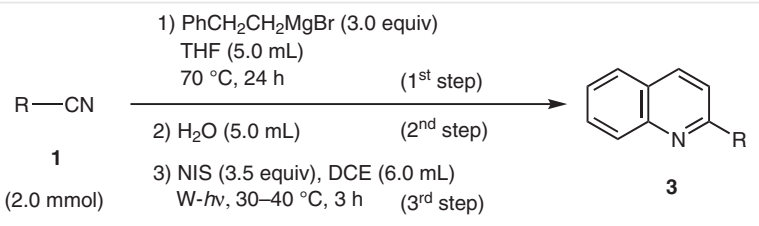<smiles>Cc1ccc(-c2ccc3ccccc3n2)cc1</smiles>

3A $82 \%$<smiles>Cc1cc(C)cc(-c2ccc3ccccc3n2)c1</smiles>

3F $77 \%$<smiles>Brc1ccc(-c2ccc3ccccc3n2)cc1</smiles>

3K $60 \%{ }^{\mathrm{c}}$<smiles>c1ccc2nc(-c3ccc4c(c3)OCO4)ccc2c1</smiles><smiles>COc1ccc(-c2ccc3ccccc3n2)cc1</smiles>

3G $75 \%$<smiles>FC(F)(F)c1ccc(-c2ccc3ccccc3n2)cc1</smiles>

3L $58 \%$<smiles>COc1ccc(-c2ccc3ccccc3n2)cc1</smiles><smiles>Cc1cccc(-c2ccc3ccccc3n2)c1</smiles>

3C $76 \%$<smiles>c1ccc(-c2ccc3ccccc3n2)cc1</smiles>

3D $84 \%$<smiles>CC(C)(C)c1ccc(-c2ccc3ccccc3n2)cc1</smiles>

3E $89 \%$<smiles>Fc1ccc(-c2ccc3ccccc3n2)cc1</smiles>

3H $83 \%$<smiles>c1ccc2cc(-c3ccc4ccccc4n3)ccc2c1</smiles>

3M $73 \%$<smiles>Cc1ccc2ccc(-c3ccccc3)nc2c1</smiles>

3R $73 \%$ d<smiles>Clc1ccc(-c2ccc3ccccc3n2)cc1</smiles>

31 $79 \%$<smiles>Brc1cccc(-c2ccc3ccccc3n2)c1</smiles>

3J $65 \%^{\mathrm{c}}$<smiles>CCCCCc1cc(-c2ccccc2)nc2ccccc12</smiles>

3U $66 \%{ }^{h}$
3Q $78 \%$<smiles>Cc1ccc(-c2cc(C)c3ccccc3n2)cc1</smiles>

3V $65 \%^{\text {h }}$<smiles>Cc1ccc(-c2nc3ccccc3cc2C)cc1</smiles>

3W $34 \%^{i}$<smiles>CC(C)c1ccc2ccccc2n1</smiles>

3X $46 \%$

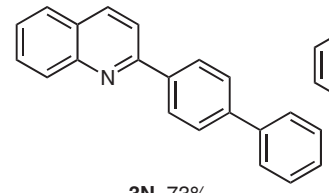
3N $73 \%$<smiles>Clc1ccc2ccc(-c3ccccc3)nc2c1</smiles>

3S $73 \%^{\mathrm{e}}$<smiles>CCC(C)c1ccc2ccccc2n1</smiles>

3Y $48 \%$<smiles>Cc1cccc(Oc2ccc(-c3ccc4ccccc4n3)cc2)c1</smiles>
$3085 \%$<smiles>COc1ccc2ccc(-c3ccccc3)nc2c1</smiles>

3T $47 \%$ f,g

Scheme 3 Preparation of 2-arylquinolines. a $2^{\text {nd }}$ step reaction was carried out for $6 \mathrm{~h}$. ${ }^{\mathrm{b}}$ Benzonitrile (1D, 10 mmol) was used. ${ }^{\mathrm{c}}$ 2-Phenylethyl bromide (4.0 equiv) was used. ${ }^{\mathrm{d}}$ Instead of 2-phenylethyl bromide, 2 -( $p$-methylphenyl)ethyl bromide (3.0 equiv) was used. ${ }^{\mathrm{e}}$ Instead of 2-phenylethyl bromide, 2 ( $p$-chlorophenyl)ethyl bromide (3.0 equiv) was used. ${ }^{f}$ Instead of 2 -phenylethyl bromide, 2 -( $p$-methoxyphenyl)ethyl bromide (3.0 equiv) was used. ${ }^{9} 3^{\text {rd }}$ step reaction was carried out at $10-20{ }^{\circ} \mathrm{C}$. ${ }^{\mathrm{h}}$ Instead of 2-phenylethyl bromide, 2 -phenyl-1-propyl bromide (3.0 equiv) was used. ${ }^{\mathrm{i}}$ Instead of 2 -phenylethyl bromide, 2-bromo-1-phenylpropane (3.0 equiv) was used.

1-phenyl-2-propylmagnesium bromide, derived from 2bromo-1-phenylpropane and magnesium, with $p$-tolunitrile under the same procedure and conditions gave 3methyl-2-(4-methylphenyl)quinoline (3W) in low yield, as addition of the Grignard reagent to $p$-tolunitrile in the $1^{\text {st }}$ reaction step does not proceed effectively due to steric hindrance.

When 2-phenylethylmagnesium bromide was treated with isobutyronitrile (1X), $\alpha$-methylbutyronitrile (1Y), and cyclohexanecarbonitrile (1Z), which are aliphatic nitriles bearing a secondary alkyl group, under the same procedure and conditions, 2-isopropylquinoline (3X), 2-sec-butylquinoline (3Y), and 2-cyclohexylquinoline (3Z), respectively, were obtained in moderate yields (Scheme 3 ). However, treatment of 2-phenylethylmagnesium bromide with propionitrile, an aliphatic nitrile bearing a primary alkyl group, under the same procedure and conditions generated 2-ethylquinoline in low yield ( $20 \%)$, due to $\alpha$-proton abstraction from propionitrile by the Grignard reagent in the $1^{\text {st }}$ reaction step. Moreover, treatment of 2-phenylethylmagnesium bromide with pivalonitrile, an aliphatic nitrile bearing a tertiary alkyl group, under the same procedure and conditions did not generate 2-tert-butylquinoline at all and, instead, 3-phenylpropionitrile was obtained in 50\% yield through the radical $\beta$-elimination of the formed iminonitrogen-centered radical. Thus, the present method is limited to the preparation of 2-arylquinolines mainly and 2-alkylquinolines bearing a secondary alkyl group. 
An opposite approach for the preparation of 2-arylquinolines with $\beta$-arylpropionitriles and arylmagnesium bromides to form ketimines $\mathbf{2}$, then the same reaction with NIS, could be proposed. However, treatment of $\beta$-phenylpropionitrile and $p$-methylphenylmagnesium bromide at $70{ }^{\circ} \mathrm{C}$ for 24 hours did not generate $p$-methylphenyl 2phenylethyl ketimine (2A) effectively due to $\alpha$-proton abstraction from $\beta$-phenylpropionitrile by $p$-methylphenylmagnesium bromide. Thus, the opposite approach (reaction of $\beta$-arylpropionitriles and $\mathrm{ArMgBr}$, followed by reaction with NIS under irradiation with a tungsten lamp) is not practical to obtain 2-arylquinolines 3.

A possible reaction pathway is shown in Scheme 4. Ketimine 2 formed from the reaction of 2-phenylethylmagnesium bromide and aromatic nitrile $\mathbf{1}\left(1^{\text {st }}\right.$ and $2^{\text {nd }}$ steps) reacts with NIS to produce $\mathrm{N}$-iodoimine $\mathbf{I}$ together with the generation of succinimide (NS). Once $N$-iodoimine $\mathbf{I}$ is formed, smooth homolytic bond cleavage of its N-I bond occurs to form imino-nitrogen-centered radical II and an iodine atom. Imino-nitrogen-centered radical II cyclizes onto the aromatic ring to form intermediate III, which is further oxidized to 3,4-dihydroquinoline IV by molecular iodine. Oxidation of 3,4-dihydroquinoline IV by NIS gives 2arylquinoline 3 via the formation of $\mathrm{N}$-iodonium compound $\mathbf{V}$ and $\mathrm{HI}$ elimination from compound $\mathbf{V I}$.

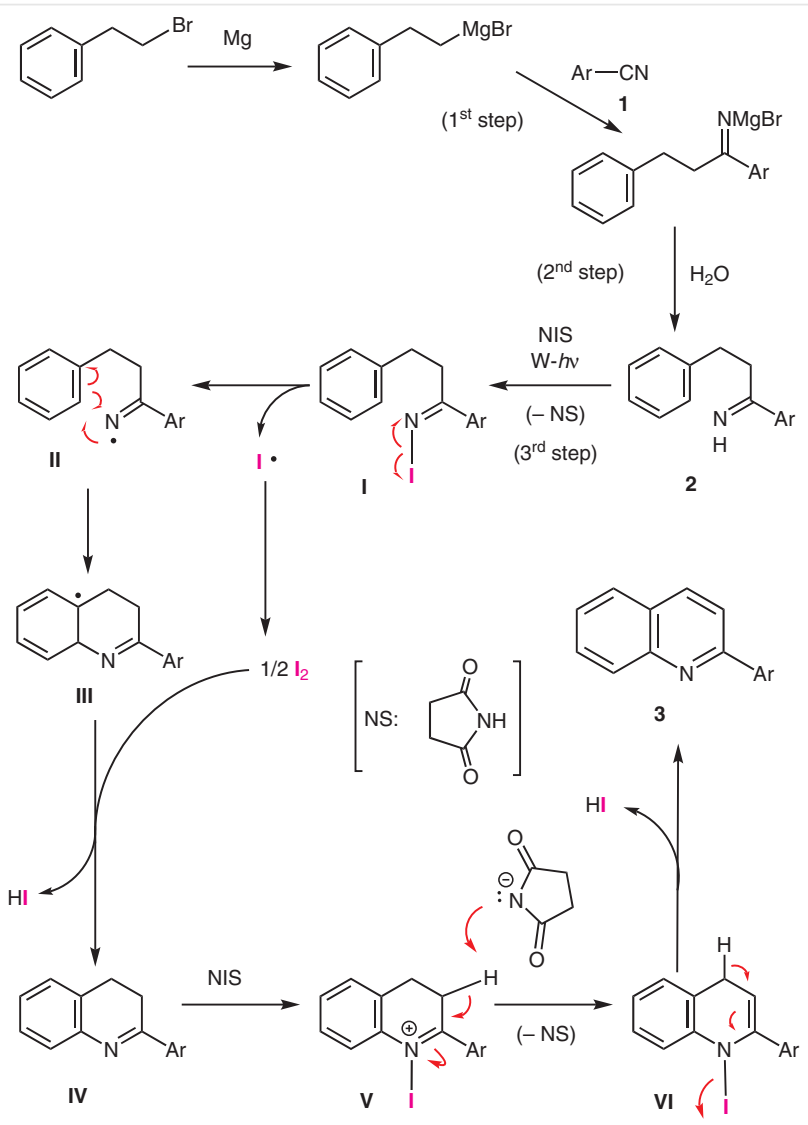

Scheme 4 Plausible reaction mechanism
In conclusion, 2-arylquinolines could be obtained smoothly and efficiently by the treatment of aryl 2-arylethyl ketimines, prepared from the reaction of 2-arylethylmagnesium bromides and aromatic nitriles, with NIS under irradiation with a tungsten lamp, through the formation of $\mathrm{N}$-iodoimines and imino-nitrogen-centered radicals, and radical cyclization onto the aromatic ring. This is a new approach for the preparation of 2-arylquinolines from commercially available 2-arylethyl bromides, aromatic nitriles, magnesium, and NIS under transition-metal-free conditions.

${ }^{1} \mathrm{H}$ NMR spectra were measured on $400 \mathrm{MHz}$ spectrometers. Data are reported as follows: chemical shift in ppm from internal tetramethylsilane on the $\delta$ scale, multiplicity ( $\mathrm{s}=$ singlet, $\mathrm{d}=$ doublet, $\mathrm{t}=$ triplet, $\mathrm{q}$ = quartet, sex = sextet, sep = septet, $\mathrm{m}=$ multiplet ), coupling constant $(\mathrm{Hz})$, integration. ${ }^{13} \mathrm{C}$ NMR spectra were measured at $100 \mathrm{MHz}$ on 400 $\mathrm{MHz}$ spectrometers. Chemical shifts are reported in ppm from the solvent resonance employed as the internal standard $\left(\mathrm{CDCl}_{3}\right.$ at 77.0 $\mathrm{ppm})$. Characteristic peaks in the IR spectra are reported in wavenumbers, $\mathrm{cm}^{-1}$. High-resolution mass spectra were recorded with Thermo Fisher Scientific Exactive Orbitrap mass spectrometers. Melting points are uncorrected. TLC was performed using $0.25 \mathrm{~mm}$ silica gel plates ( $60 \mathrm{~F} 254)$. The products were purified by column chromatography on neutral silica gel 60N (63-200 mesh).

\section{1-(4-Methylphenyl)-3-phenylpropan-1-one (2A')}

Yield: $422.1 \mathrm{mg}(94 \%) ;$ yellow oil.

IR (neat): 2959, $1676 \mathrm{~cm}^{-1}$.

${ }^{1} \mathrm{H}$ NMR $\left(400 \mathrm{MHz}, \mathrm{CDCl}_{3}\right): \delta=2.41(\mathrm{~s}, 3 \mathrm{H}), 3.06(\mathrm{t}, J=7.3 \mathrm{~Hz}, 2 \mathrm{H})$, $3.27(\mathrm{t}, J=7.0 \mathrm{~Hz}, 2 \mathrm{H}), 7.18-7.31(\mathrm{~m}, 7 \mathrm{H}), 7.85(\mathrm{~d}, J=8.2 \mathrm{~Hz}, 2 \mathrm{H})$.

${ }^{13} \mathrm{C}$ NMR $\left(100 \mathrm{MHz}, \mathrm{CDCl}_{3}\right): \delta=21.5,30.1,40.2,126.0,128.0,128.3$, 128.4, 129.2, 134.2, 141.3, 143.7, 198.7.

HRMS (APCI): $m / z[M+H]^{+}$calcd for $\mathrm{C}_{16} \mathrm{H}_{17} \mathrm{O}$ : 225.1274; found: 225.1270

\section{4-Methyl-1-phenylpentan-3-one (2X')}

Yield: $200.9 \mathrm{mg}$ (57\%); yellow oil.

IR (neat): 2968, $1705 \mathrm{~cm}^{-1}$.

${ }^{1} \mathrm{H}$ NMR $\left(400 \mathrm{MHz}, \mathrm{CDCl}_{3}\right): \delta=1.07(\mathrm{~d}, J=6.8 \mathrm{~Hz}, 6 \mathrm{H}), 2.57$ (quintet, $J=7.0 \mathrm{~Hz}, 1 \mathrm{H}), 2.77(\mathrm{t}, J=7.7 \mathrm{~Hz}, 2 \mathrm{H}), 2.89(\mathrm{t}, J=7.7 \mathrm{~Hz}, 2 \mathrm{H}), 7.17-$ $7.20(\mathrm{~m}, 3 \mathrm{H}), 7.29(\mathrm{~d}, J=7.7 \mathrm{~Hz}, 2 \mathrm{H})$.

${ }^{13} \mathrm{C}$ NMR $\left(100 \mathrm{MHz}, \mathrm{CDCl}_{3}\right): \delta=18.0,29.7,40.8,41.8,125.9,128.2$, 128.3, 141.2, 213.5 .

HRMS (APCI): $m / z[M+H]^{+}$calcd for $\mathrm{C}_{12} \mathrm{H}_{17} \mathrm{O}$ : 177.1274; found: 177.1277.

2-(4-Methylphenyl)quinoline (3A); Typical Procedure for the Preparation of 2-Arylquinolines 3 from 2-Arylethyl Bromides and Aromatic Nitriles 1

To a solution of $\mathrm{Mg}$ (160.4 mg, $6.6 \mathrm{mmol})$ in THF (3.0 mL) was added 2-phenylethyl bromide $(1110.0 \mathrm{mg}, 6.0 \mathrm{mmol})$ at room temperature under argon atmosphere. After $90 \mathrm{~min}$, the obtained Grignard reagent solution was added to a solution of $p$-tolunitrile $(\mathbf{1 A} ; 234.3 \mathrm{mg}, 2.0$ $\mathrm{mmol})$ in THF $(2.0 \mathrm{~mL})$ at room temperature. The obtained mixture was stirred for $24 \mathrm{~h}$ at $70{ }^{\circ} \mathrm{C}$ under argon atmosphere. Then, $\mathrm{H}_{2} \mathrm{O}(5.0$ 
$\mathrm{mL}$ ) was added to the mixture, and the obtained mixture was filtered through Celite. Then, the product was extracted from the filtrates with $\mathrm{CHCl}_{3}(3 \times 15.0 \mathrm{~mL})$. The organic layer was dried over $\mathrm{Na}_{2} \mathrm{SO}_{4}$. After filtration and removal of the solvent under reduced pressure, 1,2dichloroethane $(6.0 \mathrm{~mL})$ and NIS ( $1.575 \mathrm{~g}, 7.0 \mathrm{mmol})$ were added to the residue at room temperature, and the obtained mixture was stirred for $3 \mathrm{~h}$ at $30-40{ }^{\circ} \mathrm{C}$ under irradiation with a tungsten lamp $(300 \mathrm{~W})$. Sat. aq. $\mathrm{Na}_{2} \mathrm{SO}_{3}$ solution $(15.0 \mathrm{~mL})$ was added to the reaction mixture and the product was extracted with $\mathrm{CHCl}_{3}(3 \times 15.0 \mathrm{~mL})$. The organic layer was dried over $\mathrm{Na}_{2} \mathrm{SO}_{4}$. After filtration and removal of the solvent, the residue was purified by silica gel column chromatography ( $n$-hexane-EtOAc, 9:1) to give $\mathbf{3 A}$.

Yield: $361.0 \mathrm{mg}$ (82\%); white solid; $\mathrm{mp} 77-78{ }^{\circ} \mathrm{C}$.

IR (neat): 3025, 1594, $1550 \mathrm{~cm}^{-1}$.

${ }^{1} \mathrm{H} \mathrm{NMR}\left(400 \mathrm{MHz}, \mathrm{CDCl}_{3}\right): \delta=2.44(\mathrm{~s}, 3 \mathrm{H}), 7.34(\mathrm{~d}, J=7.9 \mathrm{~Hz}, 2 \mathrm{H})$, $7.51(\mathrm{td}, J=7.6,1.1 \mathrm{~Hz}, 1 \mathrm{H}), 7.72(\mathrm{td}, J=7.7,1.4 \mathrm{~Hz}, 1 \mathrm{H}), 7.82(\mathrm{~d}, J=$ $8.2 \mathrm{~Hz}, 1 \mathrm{H}), 7.87(\mathrm{~d}, J=8.6 \mathrm{~Hz}, 1 \mathrm{H}), 8.08(\mathrm{~d}, J=8.4 \mathrm{~Hz}, 2 \mathrm{H}), 8.16(\mathrm{~d}, J=$ $9.1 \mathrm{~Hz}, 1 \mathrm{H}), 8.21(\mathrm{~d}, J=8.6 \mathrm{~Hz}, 1 \mathrm{H})$.

${ }^{13} \mathrm{C}$ NMR $\left(100 \mathrm{MHz}, \mathrm{CDCl}_{3}\right): \delta=21.3,118.8(2 \mathrm{C}), 126.1,127.1,127.4$, 129.6 (3 C), 136.6, 136.8, 139.4, 148.3, 157.3.

HRMS (ESI): $m / z[M+H]^{+}$calcd for $\mathrm{C}_{16} \mathrm{H}_{14} \mathrm{~N}$ : 220.1121; found: 220.1116 .

\section{2-(2-Methylphenyl)quinoline (3B)}

Yield: $154.4 \mathrm{mg}$ (35\%); white solid; $\mathrm{mp} 76-78{ }^{\circ} \mathrm{C}$.

IR (neat): 3056, 1593, $1501 \mathrm{~cm}^{-1}$.

${ }^{1} \mathrm{H} \mathrm{NMR}\left(400 \mathrm{MHz}, \mathrm{CDCl}_{3}\right): \delta=2.42(\mathrm{~s}, 3 \mathrm{H}), 7.32-7.35$ (m, $\left.3 \mathrm{H}\right), 7.49-$ $7.51(\mathrm{~m}, 1 \mathrm{H}), 7.55(\mathrm{~d}, J=8.5 \mathrm{~Hz}, 1 \mathrm{H}), 7.57(\mathrm{dt}, J=7.6,1.1 \mathrm{~Hz}, 1 \mathrm{H})$, $7.74(\mathrm{td}, J=7.8,1.6 \mathrm{~Hz}, 1 \mathrm{H}), 7.87(\mathrm{~d}, J=8.2 \mathrm{~Hz}, 1 \mathrm{H}), 8.16(\mathrm{~d}, J=8.4 \mathrm{~Hz}$, $1 \mathrm{H}), 8.22(\mathrm{~d}, J=8.4 \mathrm{~Hz}, 1 \mathrm{H})$.

${ }^{13} \mathrm{C}$ NMR $\left(100 \mathrm{MHz}, \mathrm{CDCl}_{3}\right): \delta=20.3,122.3,126.0(2 \mathrm{C}), 126.4,126.7$, 127.5, 128.5, 129.6, 130.8, 135.9, 136.0 (2 C), 140.7, 147.9, 160.3.

HRMS (ESI): $m / z[M+H]^{+}$calcd for $\mathrm{C}_{16} \mathrm{H}_{14} \mathrm{~N}$ : 220.1121; found: 220.1121.

\section{2-(3-Methylphenyl)quinoline (3C)}

Yield: $335.0 \mathrm{mg}$ (76\%); yellow oil.

IR (neat): 3057, 1618, $1556 \mathrm{~cm}^{-1}$.

${ }^{1} \mathrm{H} \mathrm{NMR}\left(400 \mathrm{MHz}, \mathrm{CDCl}_{3}\right): \delta=2.49(\mathrm{~s}, 3 \mathrm{H}), 7.28(\mathrm{~d}, J=7.5 \mathrm{~Hz}, 1 \mathrm{H})$, $7.42(\mathrm{t}, J=7.7 \mathrm{~Hz}, 1 \mathrm{H}), 7.53(\mathrm{td}, J=7.6,1.1 \mathrm{~Hz}, 1 \mathrm{H}), 7.73(\mathrm{td}, J=7.7$, $1.4 \mathrm{~Hz}, 1 \mathrm{H}), 7.83(\mathrm{~d}, J=8.2 \mathrm{~Hz}, 1 \mathrm{H}), 7.87(\mathrm{~d}, J=8.6 \mathrm{~Hz}, 1 \mathrm{H}), 7.92(\mathrm{~d}, J=$ $7.7 \mathrm{~Hz}, 1 \mathrm{H}), 8.01(\mathrm{~s}, 1 \mathrm{H}), 8.18(\mathrm{~d}, J=8.4 \mathrm{~Hz}, 1 \mathrm{H}), 8.22(\mathrm{~d}, J=8.6 \mathrm{~Hz}, 1$ $\mathrm{H})$.

${ }^{13} \mathrm{C}$ NMR $\left(100 \mathrm{MHz}, \mathrm{CDCl}_{3}\right): \delta=21.5,119.1,124.6,126.1,127.1,127.4$, 128.2, 128.7, 129.6 (2 C), 130.1, 136.6, 138.5, 139.6, 148.2, 157.5 .

HRMS (ESI): $m / z[M+H]^{+}$calcd for $\mathrm{C}_{16} \mathrm{H}_{14} \mathrm{~N}$ : 220.1121; found: 220.1117.

\section{2-Phenylquinoline (3D)}

Yield: $346.0 \mathrm{mg}$ (84\%); white solid; $\mathrm{mp} 83-85^{\circ} \mathrm{C}$.

IR (neat): 3057, 1597, $1556 \mathrm{~cm}^{-1}$.

${ }^{1} \mathrm{H} \mathrm{NMR}\left(400 \mathrm{MHz}, \mathrm{CDCl}_{3}\right): \delta=7.47(\mathrm{tt}, J=7.2,2.1 \mathrm{~Hz}, 1 \mathrm{H}), 7.53(\mathrm{~d}, J=$ $6.4 \mathrm{~Hz}, 1 \mathrm{H}), 7.54(\mathrm{t}, J=6.9 \mathrm{~Hz}, 2 \mathrm{H}), 7.74(\mathrm{td}, J=7.8,1.5 \mathrm{~Hz}, 1 \mathrm{H}), 7.84$ $(\mathrm{d}, J=8.0 \mathrm{~Hz}, 1 \mathrm{H}), 7.89$ (d, $J=8.5 \mathrm{~Hz}, 1 \mathrm{H}), 8.16-8.19$ (m, $3 \mathrm{H}), 8.24$ (d, $J=8.5 \mathrm{~Hz}, 1 \mathrm{H}$ ).
${ }^{13} \mathrm{C}$ NMR $\left(100 \mathrm{MHz}, \mathrm{CDCl}_{3}\right): \delta=119.0,126.3,127.1,127.4,127.5$, $128.8,129.3,129.6,129.7,136.8,139.7,148.2,157.4$.

HRMS (ESI): $m / z[M+H]^{+}$calcd for $\mathrm{C}_{15} \mathrm{H}_{12} \mathrm{~N}$ : 206.0964; found: 206.0959.

\section{2-(4-tert-Butylphenyl)quinoline (3E)}

Yield: $466.3 \mathrm{mg}$ (89\%); white solid; $\mathrm{mp} 77^{\circ} \mathrm{C}$.

IR (neat): 2967, 1597, $1550 \mathrm{~cm}^{-1}$.

${ }^{1} \mathrm{H}$ NMR $\left(400 \mathrm{MHz}, \mathrm{CDCl}_{3}\right): \delta=1.38(\mathrm{~s}, 9 \mathrm{H}), 7.51(\mathrm{td}, J=7.6,1.1 \mathrm{~Hz}, 1$ H), $7.55(\mathrm{~d}, J=8.4 \mathrm{~Hz}, 2 \mathrm{H}), 7.72(\mathrm{td}, J=7.7,1.4 \mathrm{~Hz}, 1 \mathrm{H}), 7.82(\mathrm{~d}, J=8.2$ $\mathrm{Hz}, 1 \mathrm{H}), 7.87(\mathrm{~d}, J=8.6 \mathrm{~Hz}, 1 \mathrm{H}), 8.09(\mathrm{dt}, J=8.6,2.0 \mathrm{~Hz}, 2 \mathrm{H}), 8.16(\mathrm{~d}$, $J=9.1 \mathrm{~Hz}, 1 \mathrm{H}), 8.21(\mathrm{~d}, J=8.6 \mathrm{~Hz}, 1 \mathrm{H})$.

${ }^{13} \mathrm{C}$ NMR $\left(100 \mathrm{MHz}, \mathrm{CDCl}_{3}\right): \delta=21.3,34.7,118.9,125.8,126.0,127.0$, 127.2, 127.4, 129.5, 129.7, 136.6, 136.9, 148.3, 152.5, 157.5 .

HRMS (ESI): $m / z[M+H]^{+}$calcd for $\mathrm{C}_{19} \mathrm{H}_{20} \mathrm{~N}$ : 262.1590; found: 262.1586 .

\section{2-(3,5-Dimethylphenyl)quinoline (3F)}

Yield: $360.2 \mathrm{mg}$ (77\%); yellow oil.

IR (neat): 3007, 1595, $1557 \mathrm{~cm}^{-1}$.

${ }^{1} \mathrm{H} \mathrm{NMR}\left(400 \mathrm{MHz}, \mathrm{CDCl}_{3}\right): \delta=2.44(\mathrm{~s}, 6 \mathrm{H}), 7.11(\mathrm{~s}, 1 \mathrm{H}), 7.52(\mathrm{t}, J=6.9$ $\mathrm{Hz}, 1 \mathrm{H}), 7.73(\mathrm{td}, J=7.0,1.6 \mathrm{~Hz}, 1 \mathrm{H}), 7.77(\mathrm{~s}, 2 \mathrm{H}), 7.83(\mathrm{~d}, J=8.2 \mathrm{~Hz}, 1$ H), $7.87(\mathrm{~d}, J=8.6 \mathrm{~Hz}, 1 \mathrm{H}), 8.18(\mathrm{~d}, J=9.1 \mathrm{~Hz}, 1 \mathrm{H}), 8.21(\mathrm{~d}, J=8.6 \mathrm{~Hz}$, $1 \mathrm{H})$.

${ }^{13} \mathrm{C}$ NMR $\left(100 \mathrm{MHz}, \mathrm{CDCl}_{3}\right): \delta=21.4,119.2,125.4,126.1,127.1,127.4$, 129.5, 129.6, 131.0, 136.6, 138.3, 140.0, 148.2, 157.7.

HRMS (ESI): $m / z[\mathrm{M}+\mathrm{H}]^{+}$calcd for $\mathrm{C}_{17} \mathrm{H}_{16} \mathrm{~N}$ : 234.1277; found: 234.1272.

\section{2-(4-Methoxyphenyl)quinoline (3G)}

Yield: $352.9 \mathrm{mg}$ (75\%); white solid; $\mathrm{mp} 118-120^{\circ} \mathrm{C}$.

IR (neat): 3012, 1595, 1550, $1028 \mathrm{~cm}^{-1}$.

${ }^{1} \mathrm{H} \mathrm{NMR}\left(400 \mathrm{MHz}, \mathrm{CDCl}_{3}\right.$ ): $\delta=3.89(\mathrm{~s}, 3 \mathrm{H}), 7.05(\mathrm{~d}, J=8.8 \mathrm{~Hz}, 2 \mathrm{H}$ ), $7.50(\mathrm{t}, J=7.5 \mathrm{~Hz}, 1 \mathrm{H}), 7.71(\mathrm{t}, J=7.7 \mathrm{~Hz}, 1 \mathrm{H}), 7.81(\mathrm{~d}, J=8.2 \mathrm{~Hz}, 1 \mathrm{H})$, $7.84(\mathrm{~d}, J=8.6 \mathrm{~Hz}, 1 \mathrm{H}), 8.13-8.16(\mathrm{~m}, 3 \mathrm{H}), 8.19(\mathrm{~d}, J=8.6 \mathrm{~Hz}, 1 \mathrm{H})$.

${ }^{13} \mathrm{C}$ NMR $\left(100 \mathrm{MHz}, \mathrm{CDCl}_{3}\right): \delta=55.4,114.2,118.5,125.9,126.9,127.4$, $128.9,129.5$ (2 C), 132.2, 136.6, 148.3, 156.9, 160.8 .

HRMS (ESI): $m / z[\mathrm{M}+\mathrm{H}]^{+}$calcd for $\mathrm{C}_{16} \mathrm{H}_{14} \mathrm{ON}$ : 236.1070; found: 236.1066.

\section{2-(4-Fluorophenyl)quinoline (3H)}

Yield: $371.9 \mathrm{mg}$ (83\%); white solid; $\mathrm{mp} 81{ }^{\circ} \mathrm{C}$.

IR (neat): 3060, 1596, $1554 \mathrm{~cm}^{-1}$.

${ }^{1} \mathrm{H}$ NMR $\left(400 \mathrm{MHz}, \mathrm{CDCl}_{3}\right): \delta=7.20(\mathrm{~d}, J=8.8 \mathrm{~Hz}, 1 \mathrm{H}), 7.23(\mathrm{~d}, J=8.6$ $\mathrm{Hz}, 1 \mathrm{H}), 7.54(\mathrm{t}, J=7.5 \mathrm{~Hz}, 1 \mathrm{H}), 7.74(\mathrm{td}, J=7.7,1.4 \mathrm{~Hz}, 1 \mathrm{H}), 7.82-$ $7.85(\mathrm{~m}, 2 \mathrm{H}), 8.14-8.19(\mathrm{~m}, 3 \mathrm{H}), 8.23(\mathrm{~d}, J=8.6 \mathrm{~Hz}, 1 \mathrm{H})$.

${ }^{13} \mathrm{C}$ NMR $\left(100 \mathrm{MHz}, \mathrm{CDCl}_{3}\right): \delta=115.8\left(\mathrm{~d}, J_{\mathrm{C}-\mathrm{F}}=21.6 \mathrm{~Hz}\right), 118.6,126.3$, $127.1,127.5,129.4\left(\mathrm{~d}, J_{\mathrm{C}-\mathrm{F}}=8.5 \mathrm{~Hz}\right), 129.6,129.8,135.8,136.9,148.2$, $156.2,163.8\left(\mathrm{~d}, J_{\mathrm{C}-\mathrm{F}}=249.0 \mathrm{~Hz}\right)$.

HRMS (ESI): $m / z[M+H]^{+}$calcd for $\mathrm{C}_{15} \mathrm{H}_{11} \mathrm{NF}$ : 224.0870; found: 224.0867.

\section{2-(4-Chlorophenyl)quinoline (3I)}

Yield: $378.7 \mathrm{mg}$ (79\%); white solid; $\mathrm{mp} 101-102{ }^{\circ} \mathrm{C}$. IR (neat): 3056, 1588, $1552 \mathrm{~cm}^{-1}$. 
${ }^{1} \mathrm{H}$ NMR $\left(400 \mathrm{MHz}, \mathrm{CDCl}_{3}\right): \delta=7.50(\mathrm{~d}, J=8.5 \mathrm{~Hz}, 2 \mathrm{H}), 7.54(\mathrm{t}, J=8.1$ $\mathrm{Hz}, 1 \mathrm{H}), 7.74(\mathrm{t}, J=7.7 \mathrm{~Hz}, 1 \mathrm{H}), 7.84(\mathrm{~d}, J=7.9 \mathrm{~Hz}, 1 \mathrm{H}), 7.85(\mathrm{~d}, J=8.6$ $\mathrm{Hz}, 1 \mathrm{H}), 8.12-8.17(\mathrm{~m}, 3 \mathrm{H}), 8.24(\mathrm{~d}, J=8.6 \mathrm{~Hz}, 1 \mathrm{H})$.

${ }^{13} \mathrm{C}$ NMR $\left(100 \mathrm{MHz}, \mathrm{CDCl}_{3}\right): \delta=118.5,126.5,127.2,127.5,128.8$, $129.0,129.7,129.8,135.5,136.9,138.0,148.2,156.0$.

HRMS (ESI): $m / z[\mathrm{M}+\mathrm{H}]^{+}$calcd for $\mathrm{C}_{15} \mathrm{H}_{11} \mathrm{NCl}$ : 240.0575; found: 240.0573 .

\section{2-(3-Bromophenyl)quinoline (3J)}

Yield: $370.5 \mathrm{mg}(65 \%)$; white solid; $\mathrm{mp} 66-67^{\circ} \mathrm{C}$. IR (neat): 3061, 2987, 1594, $1550 \mathrm{~cm}^{-1}$.

${ }^{1} \mathrm{H}$ NMR $\left(400 \mathrm{MHz}, \mathrm{CDCl}_{3}\right): \delta=7.40(\mathrm{t}, J=7.9 \mathrm{~Hz}, 1 \mathrm{H}), 7.55(\mathrm{t}, J=7.0$ $\mathrm{Hz}, 1 \mathrm{H}), 7.59(\mathrm{~d}, J=8.8 \mathrm{~Hz}, 1 \mathrm{H}), 7.75(\mathrm{t}, J=7.0 \mathrm{~Hz}, 1 \mathrm{H}), 7.84(\mathrm{~d}, J=8.4$ $\mathrm{Hz}, 1 \mathrm{H}), 7.85(\mathrm{~d}, J=8.5 \mathrm{~Hz}, 1 \mathrm{H}), 8.08(\mathrm{~d}, J=7.7 \mathrm{~Hz}, 1 \mathrm{H}), 8.17(\mathrm{~d}, J=8.6$ $\mathrm{Hz}, 1 \mathrm{H}), 8.25(\mathrm{~d}, J=8.6 \mathrm{~Hz}, 1 \mathrm{H}), 8.35-8.36(\mathrm{~m}, 1 \mathrm{H})$.

${ }^{13} \mathrm{C}$ NMR $\left(100 \mathrm{MHz}, \mathrm{CDCl}_{3}\right): \delta=118.6,123.1,126.0,126.6,127.3$, 127.4, 129.7, 129.8, 130.3, 130.6, 132.1, 136.9, 141.6, 148.1, 155.5.

HRMS (ESI): $m / z[\mathrm{M}+\mathrm{H}]^{+}$calcd for $\mathrm{C}_{15} \mathrm{H}_{11} \mathrm{~N}^{79} \mathrm{Br}$ : 284.0069; found: 284.0070 .

\section{2-(4-Bromophenyl)quinoline (3K)}

Yield: $342.7 \mathrm{mg}$ (60\%); white solid; $\mathrm{mp} 109-110^{\circ} \mathrm{C}$.

IR (neat): 3057, 3034, 1594, $1550 \mathrm{~cm}^{-1}$.

${ }^{1} \mathrm{H} \mathrm{NMR}\left(400 \mathrm{MHz}, \mathrm{CDCl}_{3}\right): \delta=7.54(\mathrm{td}, J=7.6,1.1 \mathrm{~Hz}, 1 \mathrm{H}), 7.66(\mathrm{~d}, J=$ $8.6 \mathrm{~Hz}, 2 \mathrm{H}), 7.74(\mathrm{td}, J=7.8,1.6 \mathrm{~Hz}, 1 \mathrm{H}), 7.84(\mathrm{~d}, J=8.2 \mathrm{~Hz}, 1 \mathrm{H}), 7.85$ $(\mathrm{d}, J=8.6 \mathrm{~Hz}, 1 \mathrm{H}), 8.06(\mathrm{~d}, J=8.6 \mathrm{~Hz}, 2 \mathrm{H}), 8.16(\mathrm{~d}, J=8.6 \mathrm{~Hz}, 1 \mathrm{H}), 8.24$ (d, $J=8.6 \mathrm{~Hz}, 1 \mathrm{H})$.

${ }^{13} \mathrm{C}$ NMR $\left(100 \mathrm{MHz}, \mathrm{CDCl}_{3}\right): \delta=118.5,123.9,126.5,127.2,127.5$, $129.1,129.7,129.8,131.9,136.9,138.5,148.2,156.0$.

HRMS (ESI): $m / z[\mathrm{M}+\mathrm{H}]^{+}$calcd for $\mathrm{C}_{15} \mathrm{H}_{11} \mathrm{~N}^{79} \mathrm{Br}$ : 284.0069; found: 284.0067.

\section{2-[4-(Trifluoromethyl)phenyl]quinoline (3L)}

Yield: $318.6 \mathrm{mg}$ (58\%); white solid; $\mathrm{mp} 121-122^{\circ} \mathrm{C}$.

IR (neat): 3073, 2983, 1594, $1556 \mathrm{~cm}^{-1}$.

${ }^{1} \mathrm{H}$ NMR $\left(400 \mathrm{MHz}, \mathrm{CDCl}_{3}\right): \delta=7.58(\mathrm{t}, J=7.9 \mathrm{~Hz}, 1 \mathrm{H}), 7.75-7.80(\mathrm{~m}$, $3 \mathrm{H}), 7.86(\mathrm{~d}, J=8.2 \mathrm{~Hz}, 1 \mathrm{H}), 7.90(\mathrm{~d}, J=8.6 \mathrm{~Hz}, 1 \mathrm{H}), 8.19(\mathrm{~d}, J=8.4 \mathrm{~Hz}$, $1 \mathrm{H}), 8.27-8.30(\mathrm{~m}, 3 \mathrm{H})$.

${ }^{13} \mathrm{C} \mathrm{NMR}\left(100 \mathrm{MHz}, \mathrm{CDCl}_{3}\right): \delta=118.7,124.2\left(\mathrm{q}, J_{\mathrm{C}-\mathrm{F}}=272.5 \mathrm{~Hz}\right), 125.7$ $\left(\mathrm{d}, J_{\mathrm{C}-\mathrm{F}}=3.8 \mathrm{~Hz}\right), 126.8,127.4,127.5,127.7,129.8,129.9,131.0\left(\mathrm{q}, J_{\mathrm{C}-\mathrm{F}}=\right.$ $32.0 \mathrm{~Hz}), 137.6,142.8,148.2,155.5$.

HRMS (ESI): $m / z[\mathrm{M}+\mathrm{H}]^{+}$calcd for $\mathrm{C}_{16} \mathrm{H}_{11} \mathrm{NF}_{3}$ : 274.0838; found: 274.0835 .

\section{2-( $\beta$-Naphthyl)quinoline (3M)}

Yield: $374.3 \mathrm{mg}$ (73\%); yellow solid; $\mathrm{mp} 145-147^{\circ} \mathrm{C}$.

IR (neat): 3053, 2925, 1594, $1556 \mathrm{~cm}^{-1}$.

${ }^{1} \mathrm{H}$ NMR $\left(400 \mathrm{MHz}, \mathrm{CDCl}_{3}\right): \delta=7.52-7.57(\mathrm{~m}, 3 \mathrm{H}), 7.76(\mathrm{td}, J=7.7,1.4$ $\mathrm{Hz}, 1 \mathrm{H}), 7.86(\mathrm{~d}, J=8.0 \mathrm{~Hz}, 1 \mathrm{H}), 7.88-7.92(\mathrm{~m}, 1 \mathrm{H}), 7.99-8.02(\mathrm{~m}$, $2 \mathrm{H}), 8.05(\mathrm{~d}, J=8.6 \mathrm{~Hz}, 1 \mathrm{H}), 8.23(\mathrm{~d}, J=8.4 \mathrm{~Hz}, 1 \mathrm{H}), 8.27(\mathrm{~d}, J=8.6 \mathrm{~Hz}$, $1 \mathrm{H}), 8.38(\mathrm{dd}, J=8.7,1.7 \mathrm{~Hz}, 1 \mathrm{H}), 8.62(\mathrm{~s}, 1 \mathrm{H})$.

${ }^{13} \mathrm{C} \mathrm{NMR}\left(100 \mathrm{MHz}, \mathrm{CDCl}_{3}\right): \delta=119.1,125.0,126.3,126.7,127.1,127.2$ (2 C), 127.5, 127.7, 128.4, 128.6, 128.8, 129.7, 133.5, 133.8, 136.8, 136.9, 148.3, 157.1 .

HRMS (ESI): $m / z[\mathrm{M}+\mathrm{H}]^{+}$calcd for $\mathrm{C}_{19} \mathrm{H}_{14} \mathrm{~N}$ : 256.1121; found: 256.1118.

\section{2-(p-Biphenyl)quinoline (3N)}

Yield: $412.5 \mathrm{mg}$ (73\%); white solid; mp $173-174{ }^{\circ} \mathrm{C}$.

IR (neat): 3053, 3035, 1595, $1569 \mathrm{~cm}^{-1}$.

${ }^{1} \mathrm{H} \mathrm{NMR}\left(400 \mathrm{MHz}, \mathrm{CDCl}_{3}\right): \delta=7.31(\mathrm{td}, J=7.4,2.0 \mathrm{~Hz}, 1 \mathrm{H}), 7.49(\mathrm{t}, J=$ $7.9 \mathrm{~Hz}, 2 \mathrm{H}), 7.54$ (td, $J=7.6,1.1 \mathrm{~Hz}, 1 \mathrm{H}), 7.69(\mathrm{~d}, J=7.0 \mathrm{~Hz}, 2 \mathrm{H}), 7.75$ $(\mathrm{td}, J=7.7,1.4 \mathrm{~Hz}, 1 \mathrm{H}), 7.78(\mathrm{~d}, J=8.6 \mathrm{~Hz}, 2 \mathrm{H}), 7.85(\mathrm{~d}, J=8.2 \mathrm{~Hz}, 1 \mathrm{H})$, $7.94(\mathrm{~d}, J=8.6 \mathrm{~Hz}, 1 \mathrm{H}), 8.19(\mathrm{~d}, J=8.4 \mathrm{~Hz}, 1 \mathrm{H}), 8.24-8.28(\mathrm{~m}, 3 \mathrm{H})$.

${ }^{13} \mathrm{C}$ NMR $\left(100 \mathrm{MHz}, \mathrm{CDCl}_{3}\right): \delta=118.8,126.3,127.1,127.2,127.4$, 127.5, 127.6, 127.9, 128.8, 129.7 (2 C), 136.8, 138.5, 140.5, 142.0, $148.3,156.8$.

HRMS (ESI): $m / z[\mathrm{M}+\mathrm{H}]^{+}$calcd for $\mathrm{C}_{21} \mathrm{H}_{16} \mathrm{~N}$ : 282.1277; found: 282.1272 .

\section{2-(4-Phenoxyphenyl)quinoline (30)}

Yield: $507.3 \mathrm{mg}$ (85\%); white solid; $\mathrm{mp} 116-117^{\circ} \mathrm{C}$.

IR (neat): $3063,3038,1589 \mathrm{~cm}^{-1}$.

${ }^{1} \mathrm{H}$ NMR $\left(400 \mathrm{MHz}, \mathrm{CDCl}_{3}\right): \delta=7.08(\mathrm{dd}, J=8.6,0.9 \mathrm{~Hz}, 2 \mathrm{H}), 7.14-7.17$ $(\mathrm{m}, 3 \mathrm{H}), 7.38(\mathrm{t}, J=7.9 \mathrm{~Hz}, 2 \mathrm{H}), 7.52(\mathrm{td}, J=7.6,1.1 \mathrm{~Hz}, 1 \mathrm{H}), 7.72(\mathrm{td}$, $J=7.7,1.4 \mathrm{~Hz}, 1 \mathrm{H}), 7.81-7.86(\mathrm{~m}, 2 \mathrm{H}), 8.14-8.17$ (m, $3 \mathrm{H}), 8.21$ (d, $J=$ $8.6 \mathrm{~Hz}, 1 \mathrm{H})$

${ }^{13} \mathrm{C}$ NMR $\left(100 \mathrm{MHz}, \mathrm{CDCl}_{3}\right): \delta=118.7,118.9,119.2,123.6,126.1$, 127.0, 127.4, 129.1, 129.6, 129.7, 129.8, 134.7, 136.8, 148.3, 156.7, 156.8, 158.6.

HRMS (ESI): $m / z[\mathrm{M}+\mathrm{H}]^{+}$calcd for $\mathrm{C}_{21} \mathrm{H}_{16} \mathrm{ON}$ : 298.1226; found: 298.1223.

\section{2-(1,3-Benzodioxol-5-yl)quinoline (3P)}

Yield: $299.6 \mathrm{mg}$ (60\%); white solid; $\mathrm{mp} 85-86^{\circ} \mathrm{C}$.

IR (neat): 2992, 2900, 1595, 1486, 1247, $1037 \mathrm{~cm}^{-1}$.

${ }^{1} \mathrm{H} \mathrm{NMR}\left(400 \mathrm{MHz}, \mathrm{CDCl}_{3}\right): \delta=6.05(\mathrm{~s}, 2 \mathrm{H}), 6.95(\mathrm{~d}, J=8.2 \mathrm{~Hz}, 1 \mathrm{H}$ ), 7.51 (td, $J=7.6,1.1 \mathrm{~Hz}, 1 \mathrm{H}), 7.66$ (dd, $J=8.2,1.6 \mathrm{~Hz}, 1 \mathrm{H}), 7.69-7.75$ $(\mathrm{m}, 2 \mathrm{H}), 7.79-7.82(\mathrm{~m}, 2 \mathrm{H}), 8.13(\mathrm{~d}, J=8.6 \mathrm{~Hz}, 1 \mathrm{H}), 8.18(\mathrm{~d}, J=8.6 \mathrm{~Hz}$, $1 \mathrm{H})$.

${ }^{13} \mathrm{C}$ NMR $\left(100 \mathrm{MHz}, \mathrm{CDCl}_{3}\right): \delta=101.3,107.9,108.5,118.6,121.7$, 126.0, 127.0, 127.4, 129.5, 129.6, 134.1, 136.7, 148.1, 148.4, 148.8, 156.6 .

HRMS (ESI): $m / z[M+H]^{+}$calcd for $\mathrm{C}_{16} \mathrm{H}_{12} \mathrm{O}_{2} \mathrm{~N}$ : 250.0863; found: 250.0860 .

\section{2-[4-(Methoxymethoxy)phenyl]quinoline (30)}

Yield: $415.5 \mathrm{mg}$ (78\%); white solid; $\mathrm{mp} 64-65^{\circ} \mathrm{C}$.

IR (neat): 2988, 1595, 1495, $1249 \mathrm{~cm}^{-1}$.

${ }^{1} \mathrm{H}$ NMR (400 MHz, $\mathrm{CDCl}_{3}$ ): $\delta=3.52(\mathrm{~s}, 3 \mathrm{H}), 5.26$ (s, $2 \mathrm{H}$ ), 7.19 (d, $J=$ $8.8 \mathrm{~Hz}, 2 \mathrm{H}), 7.51(\mathrm{t}, J=6.8 \mathrm{~Hz}, 1 \mathrm{H}), 7.71(\mathrm{t}, J=7.0 \mathrm{~Hz}, 1 \mathrm{H}), 7.80-7.85$ $(\mathrm{m}, 2 \mathrm{H}), 8.12-8.15(\mathrm{~m}, 3 \mathrm{H}), 8.19(\mathrm{~d}, J=8.6 \mathrm{~Hz}, 1 \mathrm{H})$.

${ }^{13} \mathrm{C}$ NMR $\left(100 \mathrm{MHz}, \mathrm{CDCl}_{3}\right): \delta=56.1,94.3,116.4,118.6,126.0(2 \mathrm{C})$, 126.9, 127.4, 128.8, 129.6, 133.4, 136.6, 148.2, 156.8, 158.3.

HRMS (ESI): $m / z[\mathrm{M}+\mathrm{H}]^{+}$calcd for $\mathrm{C}_{17} \mathrm{H}_{16} \mathrm{O}_{2} \mathrm{~N}$ : 266.1176; found: 266.1171.

\section{7-Methyl-2-phenylquinoline (3R)}

Yield: $321.5 \mathrm{mg}$ (73\%); white solid; $\mathrm{mp} 61-62{ }^{\circ} \mathrm{C}$. IR (neat): 2987, 2901, 1596, $1550 \mathrm{~cm}^{-1}$. 
${ }^{1} \mathrm{H}$ NMR $\left(400 \mathrm{MHz}, \mathrm{CDCl}_{3}\right): \delta=2.55(\mathrm{~s}, 3 \mathrm{H}), 7.45(\mathrm{tt}, J=7.3,2.0 \mathrm{~Hz}, 1$ H), 7.50-7.59 (m, $4 \mathrm{H}), 7.84(\mathrm{~d}, J=8.6 \mathrm{~Hz}, 1 \mathrm{H}), 8.07(\mathrm{~d}, J=8.6 \mathrm{~Hz}, 1 \mathrm{H})$, $8.13-8.16(\mathrm{~m}, 3 \mathrm{H})$.

${ }^{13} \mathrm{C}$ NMR $\left(100 \mathrm{MHz}, \mathrm{CDCl}_{3}\right): \delta=21.6,119.0,126.3,127.1,127.4,128.8$, $129.1,129.4,131.9,136.1$ (2 C), 140.0, 146.8, 156.4 .

HRMS (ESI): $m / z[\mathrm{M}+\mathrm{H}]^{+}$calcd for $\mathrm{C}_{16} \mathrm{H}_{14} \mathrm{~N}$ : 220.1121; found: 220.1118 .

\section{7-Chloro-2-phenylquinoline (3S)}

Yield: $350.4 \mathrm{mg}$ (73\%); white solid; $\mathrm{mp} 102-104^{\circ} \mathrm{C}$.

IR (neat): 2987, 2901, 1594, $1547 \mathrm{~cm}^{-1}$.

${ }^{1} \mathrm{H} \mathrm{NMR}\left(400 \mathrm{MHz}, \mathrm{CDCl}_{3}\right): \delta=7.48(\mathrm{tt}, J=7.3,2.6 \mathrm{~Hz}, 1 \mathrm{H}), 7.54(\mathrm{t}, J=$ $7.5 \mathrm{~Hz}, 2 \mathrm{H}), 7.66$ (dd, $J=9.0,2.5 \mathrm{~Hz}, 1 \mathrm{H}), 7.82(\mathrm{~s}, 1 \mathrm{H}), 7.91(\mathrm{~d}, J=8.6$ $\mathrm{Hz}, 1 \mathrm{H}), 8.11(\mathrm{~d}, J=9.1 \mathrm{~Hz}, 1 \mathrm{H}), 8.15-8.17(\mathrm{~m}, 3 \mathrm{H})$.

${ }^{13} \mathrm{C}$ NMR $\left(100 \mathrm{MHz}, \mathrm{CDCl}_{3}\right): \delta=119.8,126.1,127.5,127.7,128.9$, 129.6, 130.6, 131.3, 131.9, 135.8, 139.2, 146.6, 157.6.

HRMS (ESI): $m / z[\mathrm{M}+\mathrm{H}]^{+}$calcd for $\mathrm{C}_{15} \mathrm{H}_{11} \mathrm{NCl}$ : 240.0575; found: 240.0574 .

\section{7-Methoxy-2-phenylquinoline (3T)}

Yield: $222.1 \mathrm{mg}$ (47\%); white solid; $\mathrm{mp} 116-118^{\circ} \mathrm{C}$.

IR (neat): 3005, 2936, 1557, $1120 \mathrm{~cm}^{-1}$.

${ }^{1} \mathrm{H}$ NMR $\left(400 \mathrm{MHz}, \mathrm{CDCl}_{3}\right): \delta=3.96(\mathrm{~s}, 3 \mathrm{H}), 7.10(\mathrm{~s}, 1 \mathrm{H}), 7.39$ (dd, $J=$ 9.2, $2.8 \mathrm{~Hz}, 1 \mathrm{H}), 7.44(\mathrm{t}, J=7.5 \mathrm{~Hz}, 1 \mathrm{H}), 7.52(\mathrm{t}, J=7.5 \mathrm{~Hz}, 2 \mathrm{H}), 7.84$ $(\mathrm{d}, J=8.6 \mathrm{~Hz}, 1 \mathrm{H}), 8.07(\mathrm{~d}, J=9.1 \mathrm{~Hz}, 1 \mathrm{H}), 8.11-8.15(\mathrm{~m}, 3 \mathrm{H})$.

${ }^{13} \mathrm{C} \mathrm{NMR}\left(100 \mathrm{MHz}, \mathrm{CDCl}_{3}\right): \delta=55.5,105.0,119.2,122.3,127.3,128.1$, $128.8,128.9,131.2,135.5,139.8,144.4,155.1,157.6$.

HRMS (ESI): $m / z[M+H]^{+}$calcd for $\mathrm{C}_{16} \mathrm{H}_{14} \mathrm{ON}$ : 236.1070; found: 236.1067.

\section{4-Methyl-2-phenylquinoline (3U)}

Yield: $289.9 \mathrm{mg}$ (66\%); yellow oil.

IR (neat): 2972, 2913, $1551 \mathrm{~cm}^{-1}$.

${ }^{1} \mathrm{H}$ NMR $\left(400 \mathrm{MHz}, \mathrm{CDCl}_{3}\right): \delta=2.78(\mathrm{~s}, 3 \mathrm{H}), 7.45(\mathrm{t}, J=7.5 \mathrm{~Hz}, 1 \mathrm{H})$, 7.50-7.57 (m, 3 H), 7.70-7.74 (m, 2 H), $8.01(\mathrm{~d}, J=8.2 \mathrm{~Hz}, 1 \mathrm{H}), 8.14-$ $8.19(\mathrm{~m}, 3 \mathrm{H})$.

${ }^{13} \mathrm{C}$ NMR $\left(100 \mathrm{MHz}, \mathrm{CDCl}_{3}\right): \delta=19.0,119.7,123.6,126.0,127.2,127.5$, $128.7,129.2,129.3,130.2,139.8,144.8,148.1,157.1$.

HRMS (ESI): $m / z[\mathrm{M}+\mathrm{H}]^{+}$calcd for $\mathrm{C}_{16} \mathrm{H}_{14} \mathrm{~N}$ : 220.1121; found: 220.1119 .

\section{4-Methyl-2-(4-methylphenyl)quinoline (3V)}

Yield: $305.2 \mathrm{mg}$ (65\%); yellow oil.

IR (neat): 2975, 2920, 1598, $1549 \mathrm{~cm}^{-1}$.

${ }^{1} \mathrm{H}$ NMR (400 MHz, $\left.\mathrm{CDCl}_{3}\right): \delta=2.43(\mathrm{~s}, 3 \mathrm{H}), 2.76(\mathrm{~s}, 3 \mathrm{H}), 7.32(\mathrm{~d}, J=$ $7.9 \mathrm{~Hz}, 2 \mathrm{H}$ ), 7.54 (td, $J=7.6,1.1 \mathrm{~Hz}, 1 \mathrm{H}), 7.68-7.73(\mathrm{~m}, 2 \mathrm{H}), 7.99$ (d, $J=7.3 \mathrm{~Hz}, 1 \mathrm{H}), 8.06(\mathrm{~d}, J=8.4 \mathrm{~Hz}, 2 \mathrm{H}), 8.16(\mathrm{~d}, J=7.9 \mathrm{~Hz}, 1 \mathrm{H})$.

${ }^{13} \mathrm{C}$ NMR $\left(100 \mathrm{MHz}, \mathrm{CDCl}_{3}\right): \delta=19.0,21.3,119.5,123.5,125.8,127.1$, $127.3,129.2,129.5,130.2,136.9,139.2,144.6,148.1,157.0$

HRMS (ESI): $m / z[M+H]^{+}$calcd for $\mathrm{C}_{17} \mathrm{H}_{16} \mathrm{~N}$ : 234.1277; found: 234.1275.

\section{3-Methyl-2-(4-methylphenyl)quinoline (3W)}

Yield: $160.5 \mathrm{mg}$ (34\%); yellow oil.

IR (neat): 3057, 1598, $1557 \mathrm{~cm}^{-1}$.
${ }^{1} \mathrm{H}$ NMR (400 MHz, $\left.\mathrm{CDCl}_{3}\right): \delta=2.43(\mathrm{~s}, 3 \mathrm{H}), 2.48(\mathrm{~s}, 3 \mathrm{H}), 7.30(\mathrm{~d}, J=$ $7.7 \mathrm{~Hz}, 2 \mathrm{H}), 7.49-7.53(\mathrm{~m}, 3 \mathrm{H}), 7.65(\mathrm{td}, J=7.7,1.6 \mathrm{~Hz}, 1 \mathrm{H}), 7.77$ (d, $J=8.2 \mathrm{~Hz}, 1 \mathrm{H}), 8.01(\mathrm{~s}, 1 \mathrm{H}), 8.11(\mathrm{~d}, J=8.4 \mathrm{~Hz}, 1 \mathrm{H})$.

${ }^{13} \mathrm{C}$ NMR $\left(100 \mathrm{MHz}, \mathrm{CDCl}_{3}\right): \delta=20.6,21.2,126.1,126.5,127.3,128.5$, $128.7,128.8,129.1$ (2 C), 136.5, 137.8 (2 C), 146.5, 160.4 .

HRMS (ESI): $m / z[M+H]^{+}$calcd for $\mathrm{C}_{17} \mathrm{H}_{16} \mathrm{~N}$ : 234.1277; found: 234.1274 .

\section{2-Isopropylquinoline (3X)}

Yield: $158.6 \mathrm{mg}$ (46\%); yellow oil.

IR (neat): 3059, 1601, $1561 \mathrm{~cm}^{-1}$.

${ }^{1} \mathrm{H} \mathrm{NMR}\left(400 \mathrm{MHz}, \mathrm{CDCl}_{3}\right): \delta=1.40(\mathrm{~d}, J=6.8 \mathrm{~Hz}, 6 \mathrm{H}), 3.27$ (sep, $J=$ $7.0 \mathrm{~Hz}, 1 \mathrm{H}), 7.35$ (d, $J=8.4 \mathrm{~Hz}, 1 \mathrm{H}), 7.48(\mathrm{td}, J=7.5,1.4 \mathrm{~Hz}, 1 \mathrm{H}), 7.68$ $(\mathrm{td}, J=7.8,1.6 \mathrm{~Hz}, 1 \mathrm{H}), 7.77(\mathrm{~d}, J=8.2 \mathrm{~Hz}, 1 \mathrm{H}), 8.06(\mathrm{~d}, J=8.4 \mathrm{~Hz}, 1 \mathrm{H})$, $8.09(\mathrm{~d}, J=8.6 \mathrm{~Hz}, 1 \mathrm{H})$.

${ }^{13} \mathrm{C}$ NMR $\left(100 \mathrm{MHz}, \mathrm{CDCl}_{3}\right): \delta=22.5,37.2,119.1,125.6,126.9,127.4$, $128.9,129.2,136.3,147.6,167.6$.

HRMS (ESI): $m / z[\mathrm{M}+\mathrm{H}]^{+}$calcd for $\mathrm{C}_{12} \mathrm{H}_{14} \mathrm{~N}$ : 172.1121; found: 172.1118.

\section{2-sec-Butylquinoline (3Y)}

Yield: $179.0 \mathrm{mg}$ (48\%); yellow oil.

IR (neat): 3063, 1601, $1561 \mathrm{~cm}^{-1}$.

${ }^{1} \mathrm{H}$ NMR $\left(400 \mathrm{MHz}, \mathrm{CDCl}_{3}\right): \delta=0.90(\mathrm{t}, J=7.3 \mathrm{~Hz}, 3 \mathrm{H}), 1.37(\mathrm{~d}, J=7.0$ $\mathrm{Hz}, 3 \mathrm{H}$ ), 1.72 (d·quintet, $J=15.0,7.5 \mathrm{~Hz}, 1 \mathrm{H}$ ), 1.86 (d·quintet, $J=15.0$, $7.0 \mathrm{~Hz}, 1 \mathrm{H}), 3.01(\mathrm{sex}, J=7.3 \mathrm{~Hz}, 1 \mathrm{H}), 7.31(\mathrm{~d}, J=8.4 \mathrm{~Hz}, 1 \mathrm{H}), 7.48(\mathrm{td}$, $J=7.6,1.1 \mathrm{~Hz}, 1 \mathrm{H}), 7.68(\mathrm{td}, J=7.6,1.6 \mathrm{~Hz}, 1 \mathrm{H}), 7.78(\mathrm{~d}, J=8.0 \mathrm{~Hz}, 1$ $\mathrm{H}), 8.05-8.10(\mathrm{~m}, 2 \mathrm{H})$.

${ }^{13} \mathrm{C}$ NMR $\left(100 \mathrm{MHz}, \mathrm{CDCl}_{3}\right): \delta=12.2,20.3,29.9,44.6,119.5,125.5$, $126.9,127.4,129.0,129.1,136.2,147.8,167.0$.

HRMS (ESI): $m / z \quad[M+H]^{+}$calcd for $\mathrm{C}_{13} \mathrm{H}_{16} \mathrm{~N}$ : 186.1277; found: 186.1275

\section{2-Cyclohexylquinoline (3Z)}

Yield: $179.0 \mathrm{mg}(36 \%)$; yellow oil.

IR (neat): 2924, 2850, 1600, $1502 \mathrm{~cm}^{-1}$.

${ }^{1} \mathrm{H}$ NMR $\left(400 \mathrm{MHz}, \mathrm{CDCl}_{3}\right): \delta=1.35(\mathrm{tt}, J=12.5,3.4 \mathrm{~Hz}, 1 \mathrm{H}), 1.48$ (qt, $J=13.0,3.2 \mathrm{~Hz}, 2 \mathrm{H}), 1.58-1.69(\mathrm{~m}, 2 \mathrm{H}), 1.78-1.81(\mathrm{~m}, 1 \mathrm{H}), 1.88-$ $1.92(\mathrm{~m}, 2 \mathrm{H}), 2.01-2.11(\mathrm{~m}, 2 \mathrm{H}), 2.92(\mathrm{tt}, J=12.0,3.4 \mathrm{~Hz}, 1 \mathrm{H}), 7.33$ $(\mathrm{d}, J=8.4 \mathrm{~Hz}, 1 \mathrm{H}), 7.47(\mathrm{t}, J=8.2 \mathrm{~Hz}, 1 \mathrm{H}), 7.67(\mathrm{td}, J=7.7,1.4 \mathrm{~Hz}, 1 \mathrm{H})$, $7.77(\mathrm{~d}, J=8.2 \mathrm{~Hz}, 1 \mathrm{H}), 8.05(\mathrm{~d}, J=8.5 \mathrm{~Hz}, 1 \mathrm{H}), 8.08(\mathrm{~d}, J=8.6 \mathrm{~Hz}, 1 \mathrm{H})$.

${ }^{13} \mathrm{C}$ NMR $\left(100 \mathrm{MHz}, \mathrm{CDCl}_{3}\right): \delta=26.0,26.5,32.8,47.6,119.5,125.5$, $126.9,127.4,128.9,129.1,136.2,147.7,166.8$.

HRMS (ESI): $m / z[M+H]^{+}$calcd for $\mathrm{C}_{15} \mathrm{H}_{18} \mathrm{~N}$ : 212.1395; found: 212.1393.

\section{Funding Information}

This work was supported by the Japan Society for the Promotion of Science (JSPS) KAKENHI Grant number JP15K05418.

\section{Supporting Information}

Supporting information for this article is available online at https://doi.org/10.1055/s-0039-1691642. 


\section{References}

(1) (a) Afzal, O.; Kumar, S.; Haider, M. R.; Ali, M. R.; Kumar, R.; Jaggi, M.; Bawa, S. Eur. J. Med. Chem. 2015, 97, 871. (b) Keri, R. S.; Patil, S. A. Biomed. Pharmacother. 2014, 68, 1161. (c) Gorka, A. P.; de Dios, A.; Roepe, P. D. J. Med. Chem. 2013, 56, 5231. (d) Kaur, K.; Jain, M.; Reddy, R. P.; Jain, R. Eur. J. Med. Chem. 2010, 45, 3245. (e) Chen, Y.; Fang, K.; Sheu, J.; Hsu, S.; Tzeng, C. J. Med. Chem. 2001, 44, 2374. (f) Roma, G.; Braccio, M.; Grossi, G.; Mattioli, F.; Ghia, M. Eur. J. Med. Chem. 2000, 35, 1021.

(2) (a) Foley, M.; Tilley, L. Pharmacol. Ther. 1998, 79, 55. (b) Kaminsky, D.; Meltzer, R. I. J. Med. Chem. 1968, 11, 160.

(3) Prajapati, S. M.; Patel, K. D.; Vekariya, R. H.; Panchal, S. N.; Patel, H. D. RSC Adv. 2014, 4, 24463.

(4) Kouznetsov, V. V.; Mendez, L. Y.; Gomez, C. M. Curr. Org. Chem. 2005, 9, 141.

(5) (a) Kumar, P.; Grag, V.; Kumar, M.; Verma, A. K. Chem. Commun. 2019, 55, 12168. (b) Mahato, S.; Mukherjee, A.; Santra, S.; Zyryanov, G. V.; Majee, A. Org. Biomol. Chem. 2019, 17, 7907. (c) Qiu, Y.; Niu, Y.; Wei, X.; Cao, B.; Wang, X.; Quan, Z. J. Org. Chem. 2019, 84, 4165. (d) Das, K.; Mondal, A.; Pal, D.; Srimani, D.
Org. Lett. 2019, 21, 3223. (e) Fan, Z.; Yang, S.; Peng, X.; Zhang, C.; Han, J.; Chen, J.; Deng, H.; Shao, M.; Zhang, H.; Cao, W. Tetrahedron 2019, 75, 868. (f) Li, Y.; Zhang, Q.; Xu, X.; Zhang, X.; Yang, Y.; Yi, W. Tetrahedron Lett. 2019, 60, 965. (g) Wei, W.; Teng, F.; Li, Y.; Song, R.; Li, J. Org. Lett. 2019, 21, 6285. (h) Rode, N. D.; Arcadi, A.; Nicola, A. D.; Marinelli, F.; Michelet, V. Org. Lett. 2018, 20, 5103. (i) Li, M.; Zheng, J.; Hu, W.; Li, C.; Li, J.; Fang, S.; Jiang, H.; Wu, W. Org. Lett. 2018, 20, 7245. (j) Wang, F.; Xu, P.; Wang, S.; Ji, S. Org. Lett. 2018, 20, 2204. (k) Yaragorla, S.; Pareek, A. Eur. J. Org. Chem. 2018, 1863. (l) Khaikate, O.; Meesin, J.; Pohmakotr, M.; Reutrakul, V.; Leowanawat, P.; Soorukram, D.; Kuhakarn, C. Org. Biomol. Chem. 2018, 16, 8553. (m) Mastalir, M.; Glatz, M.; Pitterauer, E.; Allmaier, G.; Kirchner, K. J. Am. Chem. Soc. 2016, 138, 15543.

(6) (a) Yin, W.; Wang, X. New J. Chem. 2019, 43, 3254. (b) Davies, J.; Morcillo, S. P.; Douglas, J. J.; Leonori, D. Chem. Eur. J. 2018, 24, 12154. (c) Jackman, M. M.; Cai, Y.; Castle, S. L. Synthesis 2017, 49, 1785. (d) Zard, S. Z. Chem. Soc. Rev. 2008, 37, 1603.

(7) Kishi, A.; Moriyama, K.; Togo, H.J. Org. Chem. 2018, 83, 11080.

(8) Naruto, H.; Togo, H. Org. Biomol. Chem. 2019, 17, 5760. 